\title{
An infinite family of non-concordant knots having the same Seifert form
}

\author{
Taehee Kim
}

\begin{abstract}
By a recent result of Livingston, it is known that if a knot has a prime power branched cyclic cover that is not a homology sphere, then there is an infinite family of non-concordant knots having the same Seifert form as the knot. In this paper, we extend this result to the full extent. We show that if the knot has nontrivial Alexander polynomial, then there exists an infinite family of non-concordant knots having the same Seifert form as the knot. As a corollary, no nontrivial Alexander polynomial determines a unique knot concordance class. We use Cochran-OrrTeichner's recent result on the knot concordance group and Cheeger-Gromov's von Neumann $\rho$-invariants with their universal bound for a 3-manifold.
\end{abstract}

Mathematics Subject Classification (2000). Primary 57M25; Secondary 57N70.

Keywords. Concordance, Seifert form, Alexander polynomial.

\section{Introduction}

We work in the topologically locally flat category. A knot is an embedding of a circle into the 3-sphere. A knot is called slice if it bounds a (locally flat) 2-disk in the 4-ball. For two knots $K_{1}$ and $K_{2}, K_{1}$ is said to be concordant to $K_{2}$ if $K_{1} \#-K_{2}$ is slice. Here the symbol \# denotes the connected sum operation and $-K$ denotes the mirror image of $K$ with reversed orientation. This is an equivalence relation. The equivalence classes (which are called the concordance classes) form an abelian group under the connected sum operation. The group is called the (classical) knot concordance group and denoted by $\mathcal{C}$. In $\mathcal{C}$, the identity is the class of slice knots. Levine [L] constructed an epimorphism $\phi: \mathcal{C} \rightarrow \mathcal{G}$ where $\mathcal{G}$ denotes the algebraic concordance group of Seifert forms modulo a certain equivalence relation. The homomorphism $\phi$ maps the concordance class represented by a knot to the algebraic concordance class represented by Seifert forms of the knot. Jiang [J] showed that the kernel of $\phi$ is infinitely generated. This implies that for each algebraic concordance class there are infinitely many (mutually) non-concordant knots whose Seifert forms represent that algebraic concordance class. But each algebraic concordance class is also represented 
by infinitely many distinct Seifert forms, and a question arises whether or not for a given Seifert form there are non-concordant knots having that Seifert form. In fact, Jiang's examples have distinct Seifert forms, hence his result does not give an answer to this question. Recently Livingston [Li] made progress and gave a partial answer under a condition on the Alexander polynomials.

Theorem ([Li, Theorem1.1]). If a knot $K$ has Seifert form $V_{K}$ and its Alexander polynomial $\Delta_{K}(t)$ has an irreducible factor that is not a cyclotomic polynomial $\phi_{n}$ with $n$ divisible by three distinct primes, then there is an infinite family $\left\{K_{i}\right\}$ of nonconcordant knots such that each $K_{i}$ has Seifert form $V_{K}$.

In the above theorem the technical condition on the Alexander polynomial is necessary since the theorem was proven by using Casson-Gordon invariants. (For Casson-Gordon invariants, refer to [CG].) More precisely, Casson-Gordon invariants are defined via characters on the first homology of prime power branched cyclic covers of knots and if every prime power branched cyclic cover of the knot has the trivial first homology then all Casson-Gordon invariants vanish. The following theorem due to Livingston shows that a knot has a prime power branched cyclic cover with nontrivial first homology under the given condition on the Alexander polynomial. In the theorem, $\Delta_{K}(t)$ denotes the Alexander polynomial of a knot $K$.

Theorem ([Li, Theorem1.2]). All prime power branched cyclic covers of a knot $K$ are homology spheres if and only if all nontrivial irreducible factors of $\Delta_{K}(t)$ are cyclotomic polynomials $\phi_{n}(t)$ with $n$ divisible by three distinct primes. All finite branched cyclic covers of $K$ are homology spheres if and only if $\Delta_{K}(t)=1$.

In addition to these results the author $[\mathrm{K}]$ proved that for each $n$ divisible by three distinct primes there exist infinitely many non-concordant knots $K_{i}$ with $\Delta_{K_{i}}(t)=$ $\left(\phi_{n}(t)\right)^{2}$ which have the same Seifert form. (In fact, in $[\mathrm{K}]$ the author showed that the knots $K_{i}$ are linearly independent in the knot concordance group.)

In this paper we extend the above results to the full extent. The main theorem is as follows.

Theorem 1.1 (Main Theorem). If a knot $K$ has Seifert form $V_{K}$ and its Alexander polynomial is not 1 , then there is an infinite family $\left\{K_{i}\right\}$ of non-concordant knots such that each $K_{i}$ has Seifert form $V_{K}$.

In fact, in the course of the proof of the main theorem, we show a stronger result that for $i \neq j$, the knots $K_{i} \#-K_{j}$ are not (1.5)-solvable. (For the definition of (1.5)solvable knots, see Section 2.) Also we note that if the rational Alexander module of the knot $K$ has a unique self-annihilating submodule with respect to the rational Blanchfield pairing, then using rationally universal solvable representations of the fundamental group of zero surgery on a knot in the 3-sphere as used in $[\mathrm{K}]$, one can construct the above knots $K_{i}$ such that they are not only mutually non-concordant 
but also linearly independent in the knot concordance group. A proof for this is not given in this paper, but one can easily prove this using arguments in [COT2, $\mathrm{K}$ ].

By Freedman's work if $\Delta_{K}(t)=1$ then $K$ is topologically slice [F, FQ]. (That is, the concordance class of $K$ is the identity in $\mathcal{C}$.) On the other hand, the main theorem implies that if $\Delta_{K}(t)$ is not 1 then there are infinitely many non-concordant knots having the Alexander polynomial $\Delta_{K}(t)$. Thus we have the following corollary.

Corollary 1.2. No nontrivial Alexander polynomial determines a unique concordance class in the knot concordance group.

In the proof of the main theorem we construct the knots $K_{i}$ by performing satellite construction on $K$. (This construction is also called genetic modification in [COT2].) This construction is briefly reviewed in the next section. To show that the $K_{i}$ are mutually non-concordant we use Cochran-Orr-Teichner's filtration of the knot concordance group in [COT1] and von Neumann $\rho$-invariants defined by Cheeger and Gromov [ChG], which were applied as knot concordance invariants first by Cochran, Orr, and Teichner in [COT1]. In particular, we use the fact that there is a universal bound for von Neumann $\rho$-invariants for a fixed 3-manifold. More precisely, for a fixed 3-manifold $M$, there exists a constant $c_{M}$ such that $\left|\rho_{\Gamma}^{(2)}(M, \psi)\right| \leq c_{M}$ for every representation $\psi: \pi_{1}(M) \rightarrow \Gamma$ where $\Gamma$ is an arbitrary group [R, Theorem 3.1.1]. We remark that in [CT] Cochran and Teichner used this fact to show that CochranOrr-Teichner's filtration of the knot concordance group is highly nontrivial, that is, $\mathcal{F}_{n} / \mathcal{F}_{n .5}$ is nontrivial for all $n \geq 2$.

\section{Preliminaries}

Throughout this paper, we use the following convention. Unless mentioned otherwise, integer coefficients are to be understood for homology groups. The zero surgery on a knot $K$ in $S^{3}$ is denoted by $M_{K}$. We use the same notation for a simple closed curve and the homology (and the homotopy) class represented by the curve. We denote $\mathbb{Q}\left[t, t^{-1}\right]$, the Laurant polynomial ring with rational coefficients, by $\Lambda$.

In this section we briefly review the machinery that will be used in the proof of the main theorem. In [COT1], Cochran, Orr, and Teichner established a filtration of the knot concordance group $\left\{\mathcal{F}_{n}\right\}_{n \in \frac{1}{2} \mathbb{N}_{0}}$ indexed by half-integers where $\mathcal{F}_{n}$ is the subgroup of (n)-solvable knots. The definition of $(n)$-solvable knots $\left(n \in \mathbb{N}_{0}\right)$ is as

follows. Recall that for a group $G, G^{(n)}$ denotes the $n^{\text {th }}$ derived group of $G$ which is defined as follows: Let $G^{(0)} \equiv G$, and inductively $G^{(n)} \equiv\left[G^{(n-1)}, G^{(n-1)}\right]$.

Definition 2.1. A knot $K$ is called (n)-solvable if $M_{K}$ bounds a spin 4-manifold $W$ such that the inclusion map $M_{K} \rightarrow W$ induces an isomorphism on the first homology 
and such that $W$ admits an (n)-Lagrangian with (n)-duals. This means that the intersection form and the self-intersection form on $H_{2}\left(W ; \mathbb{Z}\left[\pi_{1}(W) / \pi_{1}(W)^{(n)}\right]\right)$, which vanish on the $(n)$-Lagrangian, pair the $(n)$-Lagrangian and the $(n)$-duals nonsingularly and that their images together freely generate $\mathrm{H}_{2}(\mathrm{~W})$. The 4-manifold $\mathrm{W}$ is called an (n)-solution for $K$ and we say $K$ is $(n)$-solvable via $W$.

Similarly, we define (n.5)-solvable knots for $n \in \mathbb{N}_{0}$. (An (n.5)-solution $W$ is required to admit an $(n+1)$-Lagrangian with $(n)$-duals.) For more details, refer to [COT1, Definition 8.5 and Definition 8.7].

Cochran-Orr-Teichner showed that every slice knot is $(n)$-solvable for all $n$ [COT1, Remark 1.3.1]. They detect (n.5)-solvable knots, $n \in \mathbb{N}_{0}$, using von Neumann $\rho$-invariants as follows.

Theorem 2.2 ([COT1, Theorem 4.2]). Suppose that $\Gamma$ is an (n)-solvable polytorsion-free-abelian group. Let $\phi: \pi_{1}\left(M_{K}\right) \rightarrow \Gamma$ be a homomorphism. If $K$ is (n.5)-solvable via a 4-manifold $W$ over which the coefficient system $\phi$ extends, then $\rho_{\Gamma}^{(2)}\left(M_{K}, \phi\right)=0$.

We explain the terminologies in the theorem. A group $G$ is called $(n)$-solvable if $G^{(n+1)}=1$. A group $G$ is defined to be poly-torsion-free-abelian (henceforth PTFA) if it admits a normal series $1=G_{0} \triangleleft G_{1} \triangleleft \cdots \triangleleft G_{m}=G$ such that the factors $G_{i+1} / G_{i}$ are torsion-free abelian. For the von-Neumann $\rho$-invariant $\rho_{\Gamma}^{(2)}\left(M_{K}, \phi\right)$, refer to [COT1, Section 5] and [COT2, Section 2].

In fact, the target group $\Gamma$ which we will use for the proof of the main theorem is a quotient group $G / G_{r}^{(n)}$ where $G_{r}^{(n)}$ is the $n^{\text {th }}$ rational derived group of $G$ defined by Harvey $[\mathrm{H}]$ as follows. Let $G_{r}^{(0)} \equiv G$. For $n \geq 1$, define $G_{r}^{(n)} \equiv\left[G_{r}^{(n-1)}, G_{r}^{(n-1)}\right] P_{n-1}$ where

$$
P_{n-1}=\left\{g \in G_{r}^{(n-1)} \mid g^{k} \in\left[G_{r}^{(n-1)}, G_{r}^{(n-1)}\right] \text { for some } k \in \mathbb{Z}-\{0\}\right\} .
$$

The quotient $G_{r}^{(i)} / G_{r}^{(i+1)}$ is isomorphic to $\left(G_{r}^{(i)} /\left[G_{r}^{(i)}, G_{r}^{(i)}\right]\right) /\{\mathbb{Z}-$ torsion $\}$ for all $i \geq 0$ [H, Lemma 3.5]. Harvey showed the quotient $G / G_{r}^{(n+1)}$ is PTFA [H, Corollary 3.6], and one easily sees that $G / G_{r}^{(n+1)}$ is $(n)$-solvable.

To construct the knots $K_{i}$ in the main theorem we use satellite construction (or genetic modification) explained as follows. Let $K$ be a knot and $\eta$ be an unknot in $S^{3}$ which is disjoint from $K$. Let $J$ be another knot. Take the union of the exterior of $\eta$ in $S^{3}$ and the exterior of $J$ in $S^{3}$ along the common boundary (which is homeomorphic to a torus) such that a meridian of $\eta$ is identified with a longitude of $J$ and a longitude of $\eta$ with a meridian of $J$. The resulting ambient manifold is homeomorphic to $S^{3}$. The image of $K$ under this construction is denoted by $K(\eta, J)$ and we say $K(\eta, J)$ is obtained by performing satellite construction on $K$ via $\eta$ 
Vol. 80 (2005) An infinite family of non-concordant knots having the same Seifert form 151

and $J$. If we let $D$ be an embedded disk in $S^{3}$ bounded by $\eta$, then this construction is equivalent to tying all the strands of $K$ transversally passing through $D$ into $J$. For more details, refer to [COT2]. This construction can be generalized to the case when we have a trivial link $\left\{\eta_{1}, \ldots, \eta_{n}\right\}$ which is disjoint from $K$ and auxiliary knots $\left\{J_{1}, \ldots, J_{n}\right\}$ by iterating the above process. In this case the resulting knot is denoted by $K\left(\left\{\eta_{1}, \ldots, \eta_{n}\right\},\left\{J_{1}, \ldots, J_{n}\right\}\right)$.

\section{Proof of Theorem 1.1}

Let $F$ be a Seifert surface of a knot $K$ with $\Delta_{K}(t) \neq 1$ and $V_{K}$ an associated Seifert form. The Seifert surface $F$ can be thought of as a disk with $2 g$ bands where $g$ is the genus of $F$. Let $\eta^{n}, 1 \leq n \leq 2 g$, be a trivial link in $S^{3}$ which is disjoint from $F$ such that the $n^{\text {th }}$ component $\eta^{n}$ links the $n^{\text {th }}$ band of $F$ once and does not link the other bands. It is known that $\eta^{n}, 1 \leq n \leq 2 g$, generate the rational Alexander module $H_{1}\left(M_{K} ; \Lambda\right)$. (For example, see [Ro].)

By [R, Theorem 3.1.1], there exists a constant $c$ such that $\left|\rho_{\Gamma}^{(2)}\left(M_{K \#-K}, \phi\right)\right| \leq c$ for every representation $\phi: \pi_{1}\left(M_{K \#-K}\right) \rightarrow \Gamma$ where $\Gamma$ is an arbitrary group. Let $J_{1}$ be a knot with vanishing Arf invariant such that $\rho_{\mathbb{Z}}^{(2)}\left(J_{1}\right)>c$. Here $\rho_{\mathbb{Z}}^{(2)}\left(J_{1}\right)$ denotes the von Neumann $\rho$-invariant $\rho_{\mathbb{Z}}^{(2)}\left(M_{J_{1}}, \phi\right)$ where $\phi: \pi_{1}\left(M_{J_{1}}\right) \rightarrow \mathbb{Z}$ is the abelianization. Note that $\rho_{\mathbb{Z}}^{(2)}\left(J_{1}\right)$ is equal to the integral of the Levine-Tristram signatures of $J_{1}$, integrated over the circle normalized to length one [COT2, Proposition 5.1]. Inductively, we define $J_{i+1}$ to be a knot with vanishing Arf invariant such that $\rho_{\mathbb{Z}}^{(2)}\left(J_{i+1}\right)>c+2 g \cdot \rho_{\mathbb{Z}}^{(2)}\left(J_{i}\right)$. These $J_{i}$ can be easily found by taking the connected sum of suitably many even copies of a left-handed trefoil. For each $i \in \mathbb{N}$, let $J_{i}^{n}$ be a copy of $J_{i}$ for $1 \leq n \leq 2 g$. That is, $J_{i}^{n} \equiv J_{i}, 1 \leq n \leq 2 g$.

Now let $K_{i} \equiv K\left(\left\{\eta^{1}, \ldots, \eta^{2 g}\right\},\left\{J_{i}^{1}, \ldots, J_{i}^{2 g}\right\}\right)$, the knot resulting from satellite construction. Since $\eta^{n}, 1 \leq n \leq 2 g$, lie in the complement of $F$ in $S^{3}, K_{i}$ have the same Seifert form $V_{K}$ as $K$. We prove $K_{i}$ are mutually non-concordant.

Fix $i$ and $j(i<j)$, and suppose that $K_{i}$ and $K_{j}$ are concordant. That is, $K_{i} \#-K_{j}$ is slice. Observe that

$K_{i} \#-K_{j}=(K \#-K)\left(\left\{\eta^{1}, \ldots, \eta^{2 g}, \bar{\eta}^{1}, \ldots, \bar{\eta}^{2 g}\right\},\left\{J_{i}^{1}, \ldots, J_{i}^{2 g},-J_{j}^{1}, \ldots,-J_{j}^{2 g}\right\}\right)$.

Here $\bar{\eta}^{n}$ denote the mirror images of $\eta^{n}, 1 \leq n \leq 2 g$.

Let $M \equiv M_{K \#-K}$ and $M^{\prime} \equiv M_{K_{i} \#-K_{j}}$. We construct a cobordism $C$ between $M$ and $M^{\prime}$ as follows. Choose a (0)-solution $W_{i}$ for $J_{i}$. (Since $J_{i}$ has vanishing Arf invariant, it is (0)-solvable. See [COT1, Remark 1.3.2].) By doing surgery along $\pi_{1}\left(W_{i}\right)^{(1)}$, we may assume tat $\pi_{1}\left(W_{i}\right) \cong \mathbb{Z}$. Similarly, we choose a $(0)$-solution $V_{j}$ for $-J_{j}$. Let $W_{i}^{n} \equiv W_{i}$ and $V_{j}^{n} \equiv V_{j}$ for $1 \leq n \leq 2 g$. Take $M \times[0,1]$ and the disjoint union $\left(\bigsqcup_{n=1}^{2 g} W_{i}^{n}\right) \amalg\left(\bigsqcup_{n=1}^{2 g} V_{j}^{n}\right)$. To form C, for each $n$ identify the 
solid torus $S^{1} \times D^{2}$ in $\partial W_{i}^{n}=\left(S^{3} \backslash N\left(J_{i}^{n}\right)\right) \cup S^{1} \times D^{2}$ (where $N\left(J_{i}^{n}\right)$ denotes an open tubular neighborhood of $J_{i}^{n}$ in $S^{3}$ ) with a tubular neighborhood of $\eta^{n} \times\{1\}$ in $M \times\{1\}$ such that a meridian of $J_{i}^{n}$ is identified with a longitude of $\eta^{n}$ and a longitude of $J_{i}^{n}$ with a meridian of $\eta^{n}$, and identify the solid torus $S^{1} \times D^{2}$ in $\partial V_{j}^{n}=\left(S^{3} \backslash N\left(-J_{j}^{n}\right)\right) \cup S^{1} \times D^{2}$ with a tubular neighborhood of $\bar{\eta}^{n} \times\{1\}$ in $M \times\{1\}$ similarly. One sees that $\partial_{-} C=M$ and $\partial_{+} C=M^{\prime}$. Moreover one sees that $C$ is spin.

Since $K_{i} \#-K_{j}$ is slice, $K_{i} \#-K_{j}$ is (1.5)-solvable by [COT1, Remark 1.3.1]. Let $W^{\prime}$ be a (1.5)-solution for $K_{i} \#-K_{j}$. In particular, $\partial W^{\prime}=M^{\prime}$. Let $W$ be the union of $C$ and $W^{\prime}$ along their common boundary $M^{\prime}$. Hence $W$ is a 4-manifold with $\partial W=M$.

Lemma 3.1. The 4-manifold $W$, which is constructed as above, is a (1)-solution for $K \#-K$.

The proof of the above lemma is postponed. Let $\Gamma \equiv \pi_{1}(W) / \pi_{1}(W)_{r}^{(2)}$. Note that $\Gamma$ is a (1)-solvable PTFA group by [H, Corollary 3.6]. Let $\phi: \pi_{1}(W) \rightarrow \Gamma$ be the projection homomorphism. Note that $M^{\prime}, M_{J_{i}^{n}}, M_{-J_{j}^{n}}$, and $W^{\prime}$ are subspaces of $W$, hence $\phi$ can be restricted to the corresponding fundamental groups. Let $\phi_{i}^{n}$ $\left(\right.$ respectively $\left.\phi_{j}^{n}\right)$ denote $\phi$ restricted to $\pi_{1}\left(M_{J_{i}^{n}}\right)\left(\right.$ respectively $\left.\pi_{1}\left(M_{-J_{j}^{n}}\right)\right), 1 \leq n \leq$ 2g. By [COT2, Proposition 3.2],

$$
\rho_{\Gamma}^{(2)}(M, \phi)=\rho_{\Gamma}^{(2)}\left(M^{\prime},\left.\phi\right|_{\pi_{1}\left(M^{\prime}\right)}\right)+\sum_{n=1}^{2 g} \rho_{\Gamma}^{(2)}\left(M_{J_{i}^{n}}, \phi_{i}^{n}\right)+\sum_{n=1}^{2 g} \rho_{\Gamma}^{(2)}\left(M_{-J_{j}^{n}}, \phi_{j}^{n}\right) .
$$

In the above equation, $\rho_{\Gamma}^{(2)}\left(M^{\prime},\left.\phi\right|_{\pi_{1}\left(M^{\prime}\right)}\right)=0$ by Theorem 2.2 since $\left.\phi\right|_{\pi_{1}\left(M^{\prime}\right)}$ extends over (1.5)-solution $W^{\prime}$. Note that $\phi_{i}^{n}$ factors through $\pi_{1}\left(W_{i}^{n}\right)$ which is isomorphic to $\mathbb{Z}$ for each $n$. If $\phi\left(\eta^{n}\right)=e$, the identity element in $\Gamma$, then $\rho_{\Gamma}^{(2)}\left(M_{J_{i}^{n}}\right)=0$. If $\phi\left(\eta^{n}\right) \neq e$, then the image of $\phi_{i}^{n}$ is isomorphic to $\mathbb{Z}$ and $\rho_{\Gamma}^{(2)}\left(M_{J_{i}^{n}}\right)=\rho_{\mathbb{Z}}^{(2)}\left(J_{i}^{n}\right)$, which is defined in the previous section, by [COT1, Proposition 5.13]. We obtain similar results for $\rho_{\Gamma}^{(2)}\left(M_{-J_{j}^{n}}\right)$. Now let $\epsilon_{i}^{n} \equiv 0$ if $\phi\left(\eta^{n}\right)=e$ and $\epsilon_{i}^{n} \equiv 1$ otherwise, $1 \leq n \leq 2 g$. Define $\epsilon_{j}^{n}, 1 \leq n \leq 2 g$, similarly. Then we have the following equation.

$$
\rho_{\Gamma}^{(2)}(M, \phi)=\sum_{n=1}^{2 g} \epsilon_{i}^{n} \rho_{\mathbb{Z}}^{(2)}\left(J_{i}^{n}\right)-\sum_{n=1}^{2 g} \epsilon_{j}^{n} \rho_{\mathbb{Z}}^{(2)}\left(J_{j}^{n}\right) .
$$

We claim that $\epsilon_{i}^{n} \neq 0$ for some $n$ or $\epsilon_{j}^{n} \neq 0$ for some $n$. One sees that $\eta^{n}$ together with $\bar{\eta}^{n}, 1 \leq n \leq 2 g$, generate the rational Alexander module $H_{1}(M ; \Lambda)$. (This is obvious since $H_{1}(M ; \Lambda)$ is isomorphic to $H_{1}\left(M_{K} ; \Lambda\right) \oplus H_{1}\left(M_{-K} ; \Lambda\right)$.) Since $\Delta_{K}(t) \neq 1, H_{1}(M ; \Lambda)$ is not trivial. Hence $K \#-K$ has the (nontrivial) 
Vol. 80 (2005) An infinite family of non-concordant knots having the same Seifert form 153

nonsingular rational Blachfield form $B \ell: H_{1}(M ; \Lambda) \times H_{1}(M ; \Lambda) \rightarrow \mathbb{Q}(t) / \Lambda$. Let $i_{*}: H_{1}(M ; \Lambda) \rightarrow H_{1}(W ; \Lambda)$ be the homomorphism induced by the inclusion. Since $P \equiv \operatorname{Ker}\left(i_{*}\right)$ is self-annihilating by [COT1, Theorem 4.4] (that is, $P=P^{\perp}$ ) and $B \ell$ is nonsingular, $i_{*}$ is not a trivial homomorphism. Hence $i_{*}\left(\eta^{n}\right) \neq 0$ for some $n$ or $i_{*}\left(\bar{\eta}^{n}\right) \neq 0$ for some $n$ in $H_{1}(W ; \Lambda)$. Since $W$ is a (1)-solution for $K \#-K$, $H_{1}(W) \cong \mathbb{Z}$. This implies that $\pi_{1}(W)_{r}^{(1)}=\pi_{1}(W)^{(1)}$. Hence

$$
\pi_{1}(W)^{(1)} / \pi_{1}(W)_{r}^{(2)} \otimes_{\mathbb{Z}} \mathbb{Q} \cong \pi_{1}(W)^{(1)} / \pi_{1}(W)^{(2)} \otimes_{\mathbb{Z}} \mathbb{Q} \cong H_{1}(W ; \Lambda) .
$$

The first isomorphism holds by [H, Lemma 3.5]. Thus $\phi\left(\eta^{n}\right) \neq e$ or $\phi\left(\bar{\eta}^{n}\right) \neq e$ for some $n$ in $\pi_{1}(W)^{(1)} / \pi_{1}(W)_{r}^{(2)}$ which is a subgroup of $\Gamma$, and this proves the claim.

Now suppose $\epsilon_{j}^{n} \neq 0$ for some $n$. By our choice of $J_{i}$ and $J_{j}$,

$$
\rho_{\Gamma}^{(2)}(M, \phi) \leq 2 g \cdot \rho_{\mathbb{Z}}^{(2)}\left(J_{i}\right)-\rho_{\mathbb{Z}}^{(2)}\left(J_{j}\right)<-c,
$$

which is a contradiction. If $\epsilon_{j}^{n}=0$ for all $n$, then $\epsilon_{i}^{n} \neq 0$ for some $n$ by the above claim. Then

$$
\rho_{\Gamma}^{(2)}(M, \phi) \geq \rho_{\mathbb{Z}}^{(2)}\left(J_{i}\right)>c,
$$

which is also a contradiction. Therefore, to complete the proof we only need to prove Lemma 3.1 and a proof is given below.

Proof of Lemma 3.1. We follow a course of the proof for a more general case in $[\mathrm{CT}]$. Using Mayer-Vietoris sequence observe that

$$
H_{1}(M) \cong H_{1}(C) \cong H_{1}\left(M^{\prime}\right) \cong H_{1}\left(W^{\prime}\right) \cong H_{1}(W) \cong \mathbb{Z} .
$$

Again using Mayer-Vietoris sequence one sees that

$$
H_{2}(C) \cong\left(\bigoplus_{n=1}^{2 g} H_{2}\left(W_{i}^{n}\right)\right) \oplus\left(\bigoplus_{n=1}^{2 g} H_{2}\left(V_{j}^{n}\right)\right) \oplus H_{2}(M)
$$

and observe that $H_{2}(W) \cong\left(H_{2}(C) \oplus H_{2}\left(W^{\prime}\right)\right) /\left(p_{*}, q_{*}\right)\left(H_{2}\left(M^{\prime}\right)\right)$ where $p_{*}$ and $q_{*}$ are induced by inclusions $p: M^{\prime} \rightarrow C$ and $q: M^{\prime} \rightarrow W^{\prime}$, respectively. Since $H^{1}\left(W^{\prime}\right) \rightarrow H^{1}\left(M^{\prime}\right)$ is an isomorphism, $H_{3}\left(W^{\prime}, M^{\prime}\right) \rightarrow H_{2}\left(M^{\prime}\right)$ is an isomorphism by duality. Thus the homomorphism $q_{*}: H_{2}\left(M^{\prime}\right) \rightarrow H_{2}\left(W^{\prime}\right)$ is a trivial homomorphism. Observe that $H_{2}(M) \cong H_{2}\left(M^{\prime}\right) \cong \mathbb{Z}$ and they are generated by a capped-off Seifert surface of $K \#-K$ and its image under satellite construction, respectively. Moreover $p_{*}: H_{2}\left(M^{\prime}\right) \rightarrow H_{2}(C)$ maps the generator of $H_{2}\left(M^{\prime}\right)$ to the generator of $\mathrm{H}_{2}(M)$. Hence

$$
H_{2}(W) \cong\left(\bigoplus_{n=1}^{2 g} H_{2}\left(W_{i}^{n}\right)\right) \oplus\left(\bigoplus_{n=1}^{2 g} H_{2}\left(V_{j}^{n}\right)\right) \oplus H_{2}\left(W^{\prime}\right) .
$$


Observe that $\pi_{1}\left(W^{\prime}\right)^{(1)}$ maps into $\pi_{1}(W)^{(1)}$ by the homomorphism induced by the inclusion. Also $\pi_{1}\left(W_{i}^{n}\right)$ and $\pi_{1}\left(V_{j}^{n}\right)$ map into $\pi_{1}(W)^{(1)}$ by the homomorphisms induced by the inclusions since $\eta^{n}$ and $\bar{\eta}^{n}$ lie in $\pi_{1}(W)^{(1)}$ and they generate $\pi_{1}\left(W_{i}^{n}\right)$ and $\pi_{1}\left(V_{j}^{n}\right)$ (which are isomorphic to $\mathbb{Z}$ ), respectively. Now using naturality of equivariant intersection forms, one sees that (0)-Lagrangians and (0)-duals for $W_{i}^{n}$ and $V_{j}^{n}$ and a (1)-Lagrangian and (1)-duals for $W^{\prime}$ together form a (1)-Lagrangian and (1)-duals for $W$. Finally, $W^{\prime}$ has two possible spin structures, and a spin structure on $W^{\prime}$ can be chosen such that $W$ is spin. This completes the proof.

Acknowledgements. The author would like to thank Jae Choon Cha for helpful conversations.

\section{References}

[CG] A. J. Casson and C. McA. Gordon, Cobordism of classical knots. In A la recherche de la topologie perdue (L. Guilllou and A. Marin, eds.), Progr. Math. 62, Birkhäuser, Boston, MA, 1986, 181-199. Zbl 0597.57001 MR 0900252

[ChG] J. Cheeger, M. Gromov, Bounds on the von Neumann dimension of $L^{2}$-cohomology and the Gauss-Bonnet theorem for open manifolds. J. Differential Geom. 21 (1985), no. 1, 1-34. Zbl 0614.53034 MR 0806699

[COT1] T. D. Cochran, K. E. Orr, and P. Teichner, Knot concordance, Whitney towers and $L^{2}$-signatures. Ann. of Math. (2) 157 (2003), no. 2, 433-519. Zbl 02057949 MR 1973052

[COT2] T. D. Cochran, K. E. Orr, and P. Teichner, Structure in the classical knot concordance group. Comment. Math. Helv. 79 (2004), no. 1, 105-123. Zbl 02055139 MR 2031301

[CT] T. D. Cochran, P. Teichner, Knot concordance group and von Neumann $\eta$-invariants. Preprint, 2003, Rice University.

[F] M. H. Freedman, The topology of four dimensional manifolds. J. Differential Geom. 17 (1982), no. 3, 357-453. Zbl 0528.57011 MR 0679066

[FQ] M. H. Freedman and F. Quinn, Topology of 4-manifolds. Princeton Mathematical Series 39, Princeton University Press, Princeton, NJ, 1990. Zbl 0705.57001 MR 1201584

[H] S. Harvey, Higher order polynomial invariants of 3-manifolds giving lower bounds for the Thurston norm. Preprint, 2002, arXiv:math. GT/0207014, to appear in Topology.

[J] B. Jiang, A simple proof that the concordance group of algebraically slice knots is infinitely generated. Proc. Amer. Math. Soc. 83 (1981), no. 1, 189-192. Zbl 0474.57004 MR 0620010

[K] T. Kim, Filtration of the classical knot concordance group and Casson-Gordon invariants. Preprint, 2002, arXiv:math.GT/0207221, to appear in Math. Proc. Cambridge Philos. Soc.

[L] J. Levine, Knot cobordism groups in codimension two. Comment. Math. Helv. 44 (1969), 229-244. Zbl 0176.22101 MR 0246314 
Vol. 80 (2005) An infinite family of non-concordant knots having the same Seifert form 155

[Li] C. Livingston, Seifert forms and concordance. Geom. Topol. 6 (2002), 403-408 (electronic). Zbl 1009.57007 MR 1928840

[R] M. Ramachandran, Von Neumann index theorems for manifolds with boundary. J. Differential Geom. 38 (1993), no. 2, 315-349. Zbl 0787.58040 MR 1237487

[Ro] D. Rolfsen, Knots and links. Math. Lecture Ser. 7, Publish or Perish, Inc., Houston, TX, 1976, second printing 1990. Zbl 0854.57002 MR 1277811

Received December 3, 2003

Taehee Kim, Mathematics Department-MS 136, Rice University, 6100 S. Main St., Houston, TX 77005-1892, U.S.A.

E-mail: tkim@rice.edu 